\title{
Optical counterpart positions of extragalactic radio sources and connecting optical and radio reference frames ${ }^{\star}$
}

\author{
Z. Aslan ${ }^{1, \star \star}$, R. Gumerov ${ }^{2}$, W. Jin ${ }^{3}$, I. Khamitov ${ }^{1}$, N. Maigurova ${ }^{4}$, G. Pinigin ${ }^{4}$, Z. Tang ${ }^{3}$, and S. Wang ${ }^{3}$ \\ 1 TÜBİTAK National Observatory, 07058 Antalya, Turkey \\ e-mail: z.aslan@iku.edu.tr \\ 2 Kazan State University, Kremlevskaya 18, Kazan, 420008, Russia \\ e-mail: Rustem.Gumerov@ksu.ru \\ 3 Shanghai Astronomical Observatory, Nandan Road 80, Shanghai, 200030, PR China \\ e-mail: jwj@shao.ac.cn \\ ${ }^{4}$ Nikolaev Astronomical Observatory, Observatorna 1, Nikolaev, 54030, Ukraine \\ e-mail: mao@nikolaev.ua
}

Received 21 August 2009 / Accepted 14 October 2009

\begin{abstract}
We discuss the results of an investigation of astrometric positions of extragalactic radio sources from a list for the International Celestial Reference Frame. About 300 fields around extragalactic radio sources were observed during the years 2000-2003. The observations were performed mainly using two telescopes equipped with CCD cameras at TUG, Turkey (Russian-Turkish Telescope RTT150) and at YAO (1 m telescope), (Kunming, China). The mean accuracies of the measured positions are 38 mas in right ascension and 35 mas in declination. A comparison between the measured optical positions determined using the UCAC2 catalog and the radio positions from the current ICRF shows that the overall optical-minus- radio offsets are -4 and +15 mas for right ascension and declination, respectively. The formal internal errors of these mean offsets are 4 mas. The results of optical positions with respect to the reference catalogue 2MASS are also given. A search for a relation between optical and radio reference frames indicates that the orientation angles are near zero within their accuracy of about 5 mas. The link accuracy becomes 3 mas when our observations are combined with other studies.
\end{abstract}

Key words. astrometry - reference systems

\section{Introduction}

The International Celestial Reference System is the fundamental celestial reference frame that was adopted by the IAU in August, 1997. Originally, the International Celestial Refence Frame (ICRF) (Ma et al. 1998) was created and its extensions ICRF-ext1 (Ma 2001) and ICRF-ext2 (Fey et al. 2004) were later issued. The current ICRF contains Very Long Baseline Interferometry (VLBI) positions of 717 sources including 212 defining sources, 109 new sources, 294 candidate sources, and 102 additional sources. The revised coordinates of candidate and other sources are also given by Fey at al. (2004). The second realization of the ICRF will be presented at the 27th IAU General Assembly in 2009 (Ma 2008). The optical reference frame (Hipparcos Celestial Reference Frame (HCRF)) was linked to the radio (ICRF) reference frame with an accuracy of \pm 0.6 mas in position at the mean epoch 1991.25 and with an accuracy of \pm 0.25 mas per year in rotation (Kovalevsky et al. 1997). However, the accuracy of this link between HCRF

* Tables 2 and 3 giving the positions are only available in electronic form at the CDS via anonymous ftp to

cdsarc.u-strasbg.fr $(130.79 .128 .5)$ or via

http://cdsweb.u-strasbg.fr/cgi-bin/qcat?J/A+A/510/A10

$\star \star$ Present address: İstanbul Kültür University, Ataköy Yerleşkesi, 34156 Istanbul, Turkey and ICRF degrades over time because of errors in proper motions. Thus, there is a need to verify and refine the relation between the two frames using different methods and telescopes. The underlying assumption is that the centers of emission of radio sources and the optical counterpart images are coincident at the accuracy level of the optical observations. We employed a method whereby secondary reference frames are used to derive astrometric positions within small fields of view of CCD observations. Here we present determinations of the positions of the optical counterparts to extragalactic radio sources (ERS) calibrated with respect to the UCAC2 and 2MASS catalogues.

The European space astrometry mission Gaia, which should be launched by 2012, will survey about 500000 quasi stellar objects (QSO) brighter than 20th magnitude and will create an optical QSO-based celestial reference frame of higher star density, which, in turn, will provide a direct link between existing radio and optical reference frames (Mignard 2003). However, ICRF will retain its importance at least because the terrestrial reference frame is defined by VLBI observations of ERS. In the following section, our observations with mainly two telescopes are described. In Sect. 3, the reduction method and observational results are described. In Sect. 4, the relation between optical and radio frames using two methods is given. The final section summarizes the main results of determinations of optical positions of ERS and the relation between optical and radio reference frames. 


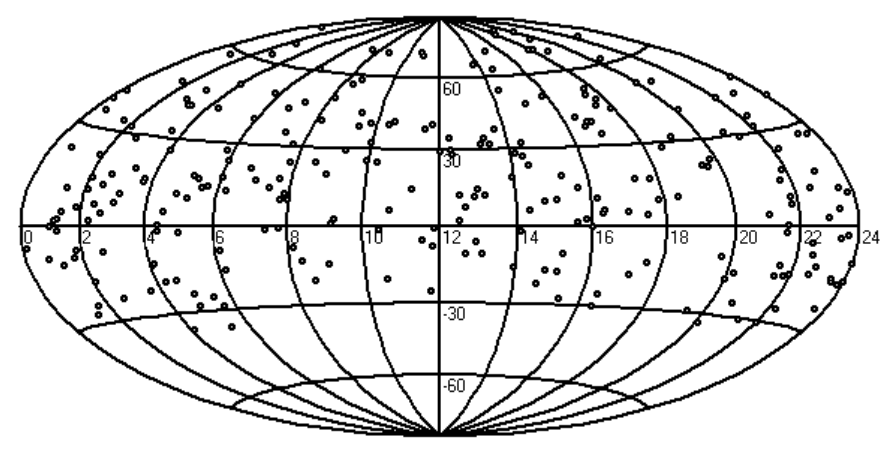

Fig. 1. Distribution of observed optical counterparts to ERS in JP over the celestial sphere.

The problem of relevant reference catalogues being used in a small field of view of CCD observations is also highlighted.

\section{Observations}

A total of about 300 optical counterparts of the ICRF radio sources were observed mostly during 2000-2003 based on a Joint Project (JP) between astronomical observatories from China, Russia, Turkey, and Ukraine (Aslan et al. 2005). Observations were carried out with two telescopes equipped with CCD cameras: Russian-Turkish Telescope (RTT150) located at the TÜBITAK National Observatory (TUG),Turkey, fully automated by Kazan State University team, and the $1 \mathrm{~m}$ telescope located at Yunnan Astronomical Observatory, China. A description of the telescopes and their detectors used for the most part of our observations are listed in Table 1. In addition, there are 8 fields around ERS obtained using the RTT150 with the CCD camera AP-47p of size $1024 \times 1024$ pixels $(\mathrm{FOV}=$ $4.0 \times 4.0$ ), and 6 fields around ERS obtained using the $2.16 \mathrm{~m}$ telescope of Beijing Astronomical Observatory ${ }^{1}$ equipped with a CCD of size $2048 \times 2048$ pixels $(\mathrm{FOV}=10.5 \times 10.5)$. At least three frames per source were typically obtained (the mean number of frames per source is about 6). All exposures were guided. Exposure time ranged from 30 to $300 \mathrm{~s}$, allowing signal-to-noise ratios for the source to be more than 3.0. The magnitude limit for the RTT150 was estimated to be $V=21$, and for the $1 \mathrm{~m}$ telescope (Yunnan Astronomical Observatory) to be $V=19$. The observations were completed for the declination range of $-40^{\circ} \leq \delta \leq 80^{\circ}$ and with uniform distribution over right ascension. Figure 1 plots the final distribution of the observed ERS in the celestial sphere.

\section{Reductions and results}

Bias, dark, and flat-field frames were also obtained during observations. CCD dark frames of the same exposure time as the object frames were applied to the raw frames, but only for frames obtained with the ST-8 camera is the dark correction significant. This CCD chip is front-illuminated and has a quantum efficiency of $30-60 \%$. It is electrically cooled to $-35^{\circ}$ from its environmental temperature. The relatively high environmental temeperature hence high CCD temperature causes a rather high dark current, and its correct calibration was critical for fainter objects (see Fig. 2).

\footnotetext{
1 Yunnan Astronomical Observatory, Beijing Astronomical Observatory and others constitute National Astronomical Observatories founded on April 25, 2001.
}

Table 1. Telescopes and CCD cameras.

\begin{tabular}{lll}
\hline \hline $\begin{array}{l}\text { Telescope } \\
\text { location }\end{array}$ & $\begin{array}{l}\text { RTT150, TUG } \\
\text { Antalya, Turkey }\end{array}$ & $\begin{array}{l}1.0 \mathrm{~m} \text { YAO } \\
\text { Kunming, China }\end{array}$ \\
\hline Coordinates $(\lambda, \varphi)$ & $+30^{\circ} 20^{\prime},+36^{\circ} 49^{\prime}$ & $+102^{\circ} 47^{\prime},+25^{\circ} 02^{\prime}$ \\
Height, m & 2500 & 1000 \\
$D(\mathrm{~mm}) / F(\mathrm{~mm})$ & $1500 / 11600$ & $1000 / 13000$ \\
CCD & $\mathrm{ST} 8^{a}$, Andor DW436 & TI \\
Size, pix. & $1530 \times 1020,2048 \times 2048$ & $1024 \times 1024$ \\
Pixel, mkm & $9 \times 9,13.5 \times 13.5$ & $24 \times 24$ \\
Scale, arcsec/pix & $0.16,0.24$ & 0.37 \\
FOV, arcmin & $4.1 \times 2.7,8.2 \times 8.2$ & $6.5 \times 6.5$ \\
\hline
\end{tabular}

Notes. ${ }^{(a)}$ Used in binning mode.

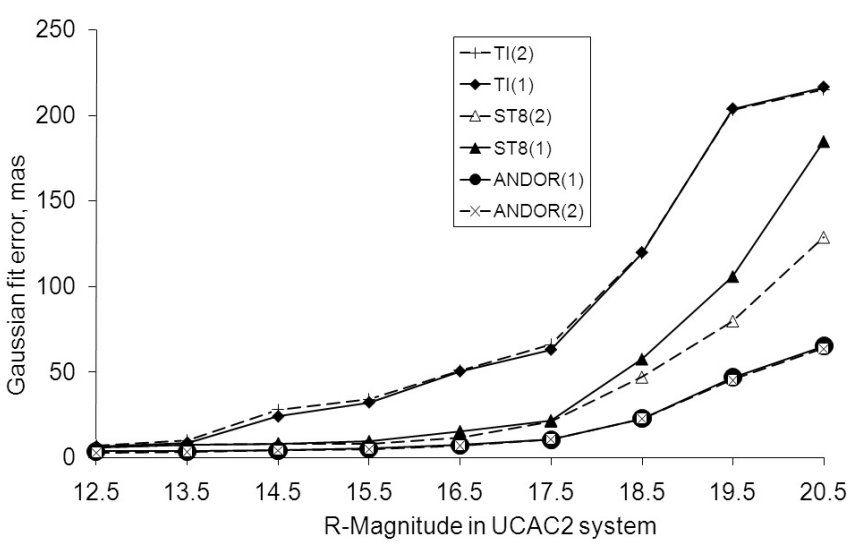

Fig. 2. Gaussian fit errors plotted versus magnitude in UCAC2 system for cameras Andor (filled circle and cross), ST-8 (open and filled triangles), and for TI (filled rectangle and plus sign). (1): with dark calibration, (2): without dark calibration.

Both the Andor CCD camera at RTT150 and TI camera at $1 \mathrm{~m}$ telescope have low noise levels. No dark current effect is found when applying dark-field at the precision of measured coordinates. The APEX II image processing package was used for processing observational data (Kouprianov 2008). All frames were reduced individually. The processing of the CCD images included the detection of star-like objects, measuring the positions $(x, y)$ of their centers, and the reduction to obtain tangential and equatorial coordinates. To derive $x, y$ coordinates of the image centers, a two-dimensional Gaussian model was applied to the image profile fits. A linear model was adopted to transform the measured CCD coordinates $(x, y)$ to tangential coordinates $(\xi, \eta)$ in a standard astrometric "plate" reduction given by

$\xi=a x+b y+c$

$\eta=d x+e y+f$.

Unweighted least-squares adjustments were performed for the standard astrometric reduction with the available reference stars. No corrections for apparent displacements, such as differential refraction, were applied, which is justified owing to the small field of view of the CCD frames. One of the main problems in astrometric reduction in small fields is the absence of reference catalogues with precise positions and proper motions. In small CCD fields, one cannot use these well-known catalogues with low star density as Hipparcos, TYCHO, or TYCHO2. The 
first reduction of our observational data was achieved using USNO catalogues (version USNO A2.0 and USNO B1.0) as reference catalogues. The results of the reduction with reference stars from USNO B1.0 catalogue show large systematic errors of about 200 mas in declination. Because of their low precision, the optical stellar positions from these catalogues cannot be used to refine the link parameters between the radio and optical systems. The UCAC2 and 2MASS (Zacharias et al. 2004; Cutri et al. 2003) are more accurate catalogues, which have enabled us to partly re-process the available observational data. But it should be noted that the UCAC2 has insufficient star density to be used as a reference catalogue in small CCD fields and the distribution is not over the whole celestial sphere, only until $48^{\circ}-52^{\circ}$ in northern declination. On the other hand, the 2MASS catalogue has no proper motions. But the mean epochs of the 2MASS catalogue and our optical observations are very close to 2000, which is why we use 2MASS here as a reference catalogue for the precise reduction of optical positions. The optical position results obtained using reference stars from the UCAC2 and 2MASS catalogues are discussed below.

\subsection{Final optical postions}

Tables 2 and 3 provide the optical positions of the ERS sources obtained using the UCAC2 and 2MASS as reference catalogues, respectively. (The errors in right ascension always include a $\cos \delta$ factor.) The Cols. 2 and 3 to 9 indicate the optical positions and standard errors in right ascension and declination, respectively, where $E r_{\alpha}$ and $E r_{\delta}$ (Cols. 5 and 9) are the formal standard (measuring) errors calculated from the scatter of the individual optical positions about the mean optical position determined from all the CCD frames of the ERS concerned. Columns 10 and 11 give the optical-minus-radio position offsets $(\mathrm{O}-\mathrm{R})$ in right ascension and declination $(\Delta \alpha \cos \delta, \Delta \delta)$ (radio positions are taken from Fey et al. 2004). Columns 12 and 13 provide the the dispersions in right ascension and declination $\left(\sigma_{\alpha}, \sigma_{\delta}\right)$ about the solution for the plate constants and are thus a measure of the accuracy of the plate transformation (1)-(2). Columns 14 and 15 are, respectively, the number of reference stars $\left(N_{\mathrm{st}}\right)$ and number of frames $\left(N_{\mathrm{fr}}\right)$ used in the reduction. The last two columns are the place of observation and the mean epoch of observation. The 130 positions of 126 ERS were obtained using the UCAC2 catalogue, and the 182 positions of 171 ERS were obtained using the 2MASS catalogue. Unfortunately, a sizeable part of the CCD frames could not be reduced because the number of reference stars was too small (less than 4) or the optical counterpart to the ERS was too faint, or totally absent, in the CCD frame. Optical positions and optical-minus-radio positional differences $(\Delta \alpha \cos \delta, \Delta \delta)$ given in Tables 2 and 3 are the unweighted means of individual differences. It should be noted that large standard errors in the optical positions can be explained not by the number of reference stars but by the faintness of the optical counterparts to ERS, technical problems, and atmospheric influence. Some fraction (less than 20\%) of these observations were included in the calculations of the rotational parameters with weight of 0.5 .

\subsection{Analysis of the diferences between radio and optical positions}

The histograms in Figs. 3 and 4 show the distribution of the differences $\mathrm{O}-\mathrm{R}$ obtained with UCAC2 and 2MASS as reference catalogues in right ascension and declination, respectively. For the 130 final O-R differences referred to the UCAC2

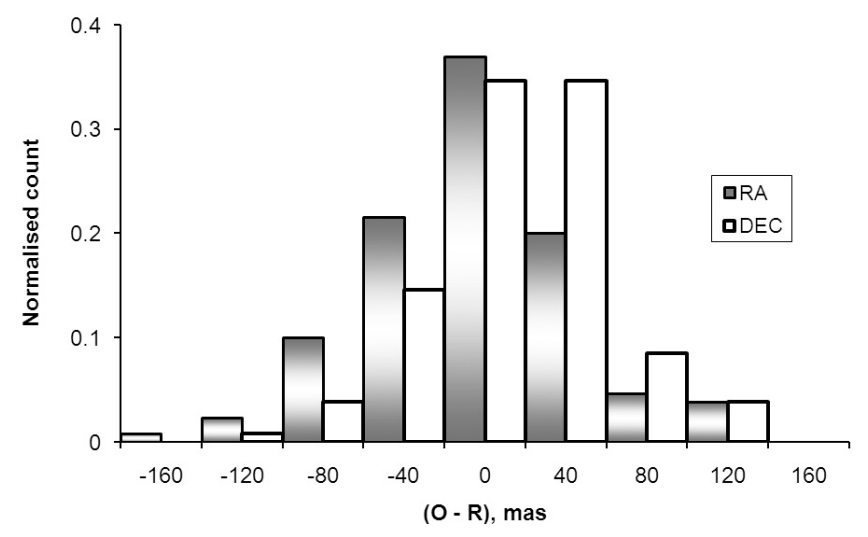

Fig. 3. Differences $(\mathrm{O}-\mathrm{R})$ distribution referred to UCAC2.

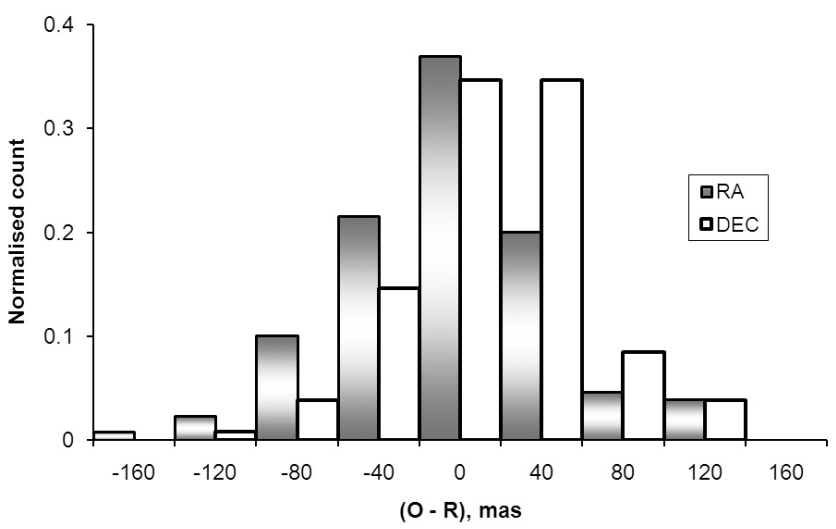

Fig. 4. Differences $(\mathrm{O}-\mathrm{R})$ distribution referred to $2 \mathrm{MASS}$.

Table 4. Mean values of $(\mathrm{O}-\mathrm{R})$ with their errors.

\begin{tabular}{cccc}
\hline \hline Reference catalog & $\Delta \alpha \cos \delta$, mas & $\Delta \delta$, mas & $N$ \\
\hline UCAC2 & $-4 \pm 5$ & $15 \pm 4$ & 130 \\
2MASS & $9 \pm 6$ & $27 \pm 6$ & 182 \\
\hline
\end{tabular}

catalogue, only 2 have absolute values of more than 150 mas. The sources concerned are $0010+405$ and $2048+312$. For the 182 final O-R differences referred to the 2MASS catalogue, 3 have absolute values of more than 250 mas; the sources concerned are $0039+230,1354+195$, and $1435+638$.

The final statistics of our results are given in Table 4, which displays the mean values of optical-minus-radio differences and their errors. Figures 5 and 6 display the averaged optical-minusradio differences with respect to the UCAC2 and 2MASS catalogues (for 124 common ERS) as a function of right ascension and declination.

As judged by their errors, no clear systematic dependence of these $(\mathrm{O}-\mathrm{R})$ differences on either right ascension or declination is shown in these figures, except perhaps a small steady increase in $(\mathrm{O}-\mathrm{R})_{\alpha}$ in the declination zone $-5^{\circ} \leq \delta \leq 30^{\circ}$, similar for both UCAC2 and 2MASS. There is also a positive offset of $(\mathrm{O}-\mathrm{R})_{\delta}$ (see Table 5 and Sect. 4), which is again similar for UCAC2 and 2MASS, but the offset for UCAC2 being systematically more positive in the right ascension zone $10^{\mathrm{h}}<\alpha<24^{\mathrm{h}}$. Further study may clarify whether these regional differences are real. Nevertheless, as the $\mathrm{O}-\mathrm{R}$ differences for the two catalogues are small and very similar,we may conclude that it is possible to 

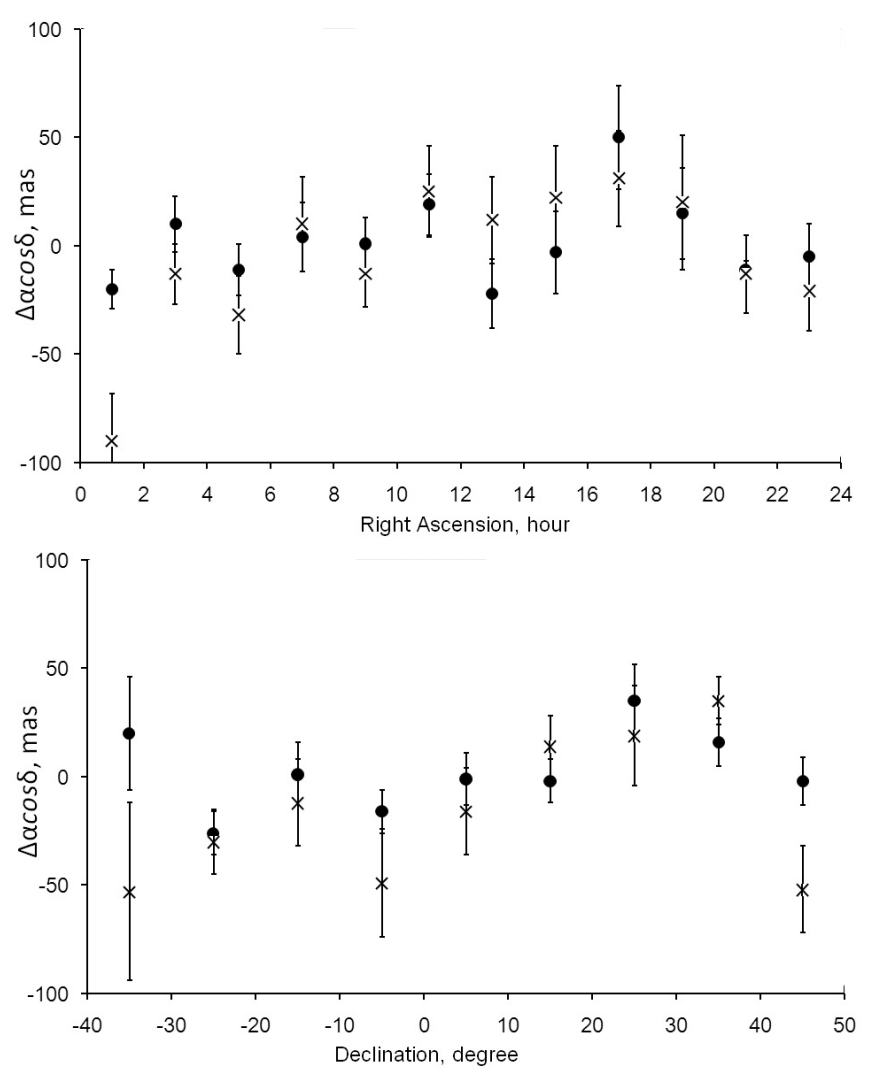

Fig. 5. Differences $(\mathrm{O}-\mathrm{R})$ in right ascension as a function of right ascension and declination (circles UCAC2 data, crosses 2MASS data) for 124 common ERS.

use 2MASS data as a reference catalogue of higher star density for astrometric reduction in a small field of the celestial sphere.

\section{The relation between the optical and radio frames}

The relative orientation between the two celestial reference frames is obtained by computing three differential rotations about the coordinate axes using the $(\mathrm{O}-\mathrm{R})$ position differences. There are several methods of representing differences between the two catalogues. Here we used a method of simple rotation and a method of rotation with bias parameter between the equators of the coordinate systems. Following the method used by Arias et al. (1988), differences between the two coordinate systems can be represented by

$$
\begin{aligned}
& \Delta \alpha_{(\mathrm{O}-\mathrm{R})} \cos \delta=\varpi_{x} \sin \delta \cos \alpha+\varpi_{y} \sin \delta \sin \alpha-\varpi_{z} \cos \delta \\
& \Delta \delta_{(\mathrm{O}-\mathrm{R})}=-\varpi_{x} \sin \alpha+\varpi_{y} \cos \alpha,
\end{aligned}
$$

where $\varpi_{x}$ is a rotation about the axis $x\left(\alpha=0^{\mathrm{h}}, \delta=0^{\circ}\right)$, $\varpi_{y}$ about the axis $y\left(\alpha=6^{\mathrm{h}}, \delta=0^{\circ}\right)$, and $\varpi_{z}$ about the direction $z\left(\delta=90^{\circ}\right)$. Taking into account all the remarks made above, the angles values were determined from available observations. Table 5 provides the results of weighted least-squares adjustment using our $(\mathrm{O}-\mathrm{R})$ differences from the UCAC2 set, data from the reference paper given by Assafin et al. (2003), and both sets of data combined. In the last column, $\sigma$ is the standard error in the least squares adjustment. For $(\mathrm{O}-\mathrm{R})$ differences with standard errors larger than the mean value of one standard deviation, we
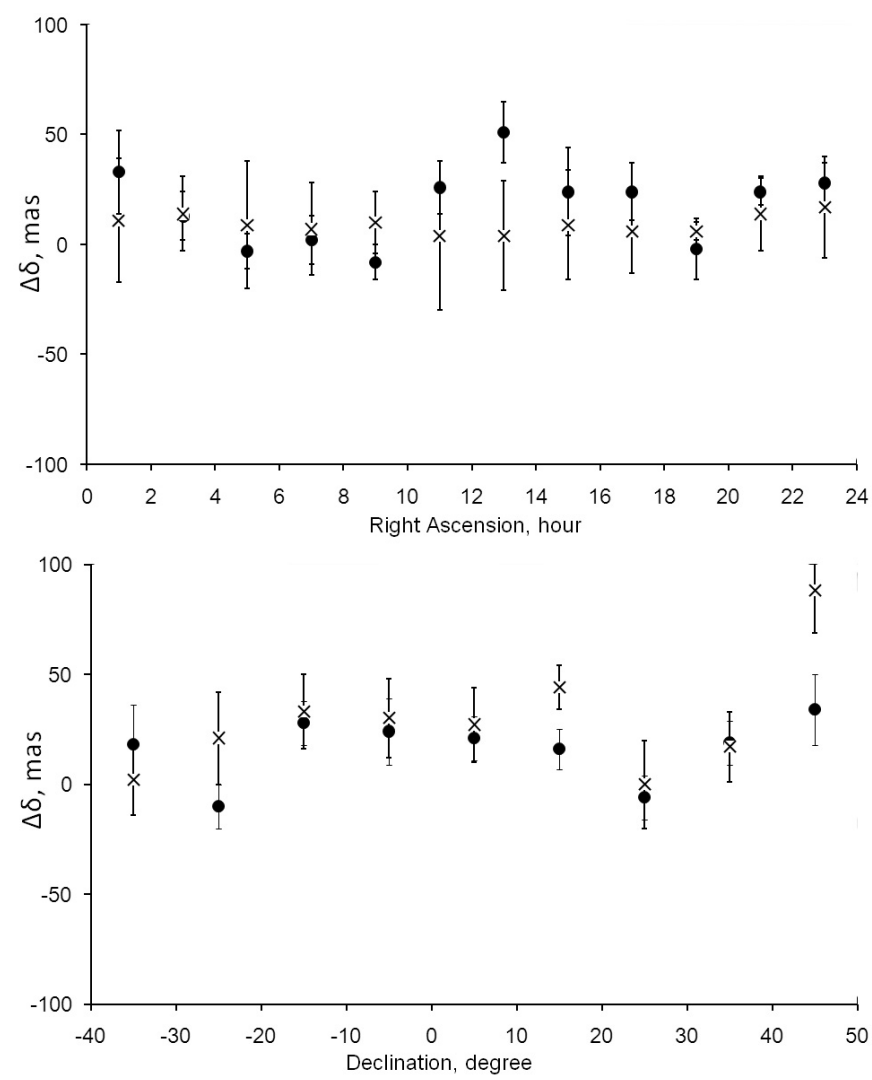

Fig. 6. Differences $(\mathrm{O}-\mathrm{R})$ in declination as a function of right ascension and declination (circles UCAC2 data, crosses 2MASS data) for 124 common ERS.

assign a weight of 0.5 . We note that the total number of such (O-R) differences is not more than $20 \%$. The results in Table 6 were derived by using the following system of equations:

$$
\begin{aligned}
& \Delta \alpha_{(\mathrm{O}-\mathrm{R})} \cos \delta=\varpi_{x} \sin \delta \cos \alpha+\varpi_{y} \sin \delta \sin \alpha-\varpi_{z} \cos \delta \\
& \Delta \delta_{(\mathrm{O}-\mathrm{R})}=-\varpi_{x} \sin \alpha+\varpi_{y} \cos \alpha+B_{0},
\end{aligned}
$$

where $\varpi_{x}, \varpi_{y}$, and $\varpi_{z}$, represent small angles around the axes $x$, $y$, and $z$, respectively, and $B_{0}$ is the bias parameter between the principal planes of the compared frames. The three rotational parameters and the parameter $B_{0}$ were determined simultaneously from available observations by least squares. Tables 5 and 6 show the standard errors of the mean values of optical-minusradio angles to be about 5 mas obtained from data in our Joint Project, and to be about 3 mas obtained from data in the work of Assafin and coworkers (Assafin et al. 2003). We note the significant difference of $27 \pm 5$ between the values of $B_{0}$ in the first two lines of Table 6. Since our observations were acquired for the northern hemisphere and the observations of Assafin et al. mostly refer to the southern hemisphere, this difference could be explained by different systematic errors in the reference catalogue UCAC2 in the respective regions. The value of $B_{0}$ from the combined data in the last line of Table 5 does not differ significantly from zero.

\section{Conclusions}

We have determined the optical positions of optical counterparts to the ERS with an accuracy level of 40 mas (for a 
Table 5. Optical-radio rotational parameters.

\begin{tabular}{lccccc}
\hline \hline Source & $\begin{array}{c}\varpi_{x} \\
\text { mas }\end{array}$ & $\begin{array}{c}\varpi_{y} \\
\text { mas }\end{array}$ & $\begin{array}{c}\varpi_{z} \\
\text { mas }\end{array}$ & $N$ & $\begin{array}{c}\sigma \\
\text { mas }\end{array}$ \\
\hline $\mathrm{JP}^{1}$ & $-0.2 \pm 5.8$ & $7.2 \pm 5.5$ & $7.0 \pm 4.5$ & 130 & 43 \\
$\mathrm{CTIO}^{2}$ & $-1.1 \pm 3.6$ & $1.3 \pm 3.0$ & $6.7 \pm 2.4$ & 172 & 31 \\
$\mathrm{JP}^{+} \mathrm{CTIO}^{3}$ & $-0.4 \pm 3.2$ & $3.5 \pm 2.8$ & $6.8 \pm 2.3$ & 302 & 37 \\
\hline
\end{tabular}

Notes. (1) JP-Joint Projects, data from this work; (2) data from work Assafin et al. (2003); ${ }^{(3)}$ joint Project data and data from work Assafin et al. (2003).

Table 6. Optical-radio rotational parameters.

\begin{tabular}{lcccc}
\hline \hline Source & $\begin{array}{c}\varpi_{x} \\
\text { mas }\end{array}$ & $\begin{array}{c}\varpi_{y} \\
\text { mas }\end{array}$ & $\begin{array}{c}\varpi_{z} \\
\text { mas }\end{array}$ & $\begin{array}{c}B_{0} \\
\text { mas }\end{array}$ \\
\hline $\mathrm{JP}^{1}$ & $1.7 \pm 5.8$ & $1.6 \pm 5.7$ & $6.9 \pm 4.4$ & $13.4 \pm 4.5$ \\
$\mathrm{CTIO}^{2}$ & $3.8 \pm 3.4$ & $1.3 \pm 2.8$ & $6.8 \pm 2.3$ & $-14.0 \pm 2.3$ \\
$\mathrm{JP}^{2} \mathrm{CTIO}^{3}$ & $-1.0 \pm 3.2$ & $4.1 \pm 2.8$ & $6.8 \pm 3.3$ & $-3.9 \pm 2.2$ \\
\hline
\end{tabular}

Notes. (1) JP-Joint Projects, data from this work; (2) data from work Assafin et al. (2003); ${ }^{(3)}$ joint Project data and data from work Assafin et al. (2003).

single position). The optical positions of the 126 ERS in the declination zone $-30^{\circ} \leq \delta \leq 50^{\circ}$ were measured with respect to the UCAC2, which was used as a reference catalogue and positions of $171 \mathrm{ERS}$ in the declination zone $-40^{\circ} \leq \delta \leq 80^{\circ}$ were measured with respect to $2 \mathrm{MASS}$ reference catalogue. The mean offsets between the ICRF radio positions and our positions relative to the UCAC2 catalogue are 4 and +15 mas in right ascension and declination, respectively. The observed optical-minus-radio position differences show no regional systematic errors within the accuracy of the catalogues used. Using the UCAC2 as a reference catalogue in determining the astrometry of a small CCD field has its limitation because of its low star density (limiting magnitude $R \approx 16.5$ ) and its northern declination limit at about $\delta \leq 48^{\circ}$ or in several zones $\delta \leq 52^{\circ}$. Optical positions of the ERS determined with respect to UCAC2 and 2MASS have shown no large systematic differences between these two catalogues. Although the 2MASS catalogue has no proper motions, we note that we are able to use it as a reference catalogue because its epoch is close to the epoch of our observations. We note in passing that this absence of proper motions makes it impossible to use 2MASS catalogue as a reference when seeking high astrometry accuracy and that a higher accuracy catalogue of higher star density is urgently required. The orientation angles between the two systems are close to zero within their accuracy of 5 mas. This result indicates that the link between HCRF and ICRF at the mean epoch of our observations is good enough to consider UCAC2 and 2MASS as similar systems. Our reduction with different sets of observational data has shown that the accuracy of the parameters relating the two catalogues improves as the available number of ERS becomes larger.

Acknowledgements. We thank TÜBİTAK National Observatory (TUG), Turkey, Moscow Space Research Institute, and Kazan State University for partial supports in using RTT150 (Russian-Turkish 1.5-m telescope in Antalya) with project number (TUG proje No. 998). Part of this work was supported by the Russion Foundation of Basic Research (grants No. 02-02-17076 and 08-02000704), and by the National Natural Science Foundation of China (grants Nos. 10673026, 10878022 and 10873014). Authors express their thanks to the collaborators of the RI Nikolaev Astronomical Observatory M. Martynov and Bondarchuk for useful participation in the processing for this paper. We thank Dr. Francois Mignard for his constructive criticism. We also thank the language editor, Dr. Claire Halliday, for her suggestions which improved the text.

\section{References}

Archinal, B. A., Arias, E. F., Gontier, A. M., \& Mercuri Moreau, C. 1997, IERS Technical Note 23, P.II11

Arias, F., Feissel, M., \& Lestrade, J. F. 1988, A\&A, 199, 357

Aslan, Z., Gumerov, R., Jin, W., et al. 2005, in Kinemat. Phys. Celest. Bodies Suppl. Ser., 5, 333

Assafin, M., Zacharias, N., Rafferty, T. J., et al. 2003, AJ, 125, 2728

Cutri, R. M., et al. 2003, The IRSA 2MASS All-Sky Point Source Catalog, NASA/IPAC

Fey, A. L., Ma, C., Arias, E. F., et al. 2004, AJ, 127, 3587

Kouprianov, V. 2008, Adv. Space Res., 41, 1029

Lindegren, L., \& Kovalevsky, J. 1995, A\&A, 304, 189

Ma, C. 2001, In Proceedings of the 15th Working Meeting on European VLBI September 07-08, ed. D. Behrend, \& A. Rius, 187

Ma, C. 2008, in A Giant Step: From Milli- to Micro-Arcsecond Astrometry, ed. W. J. Jin, I. Platais, \& M. A. C. Perryman (Cambridge: Cambridge University Press), IAU Symp., 248, 337

Ma, C., Arias, E. F., Eubanks, T. M., et al. 1998, AJ, 116, 516

Mignard, F. 2003, in IAU 25 joint Discussion, 16, 133

Zacharias, N., Urban, S. E., Zacharias, M. I., et al. 2004, AJ, 127, 3043 